Proyecciones Journal of Mathematics

Vol. 37, No 2, pp. 295-304, June 2018.

Universidad Católica del Norte

Antofagasta - Chile

\title{
Upper double monophonic number of a graph
}

\author{
A. P. Santhakumaran \\ Hindustan Institute of Technology and Science, India \\ and \\ T. Venkata Raghu \\ Sasi Institute of Technology and Engineering, India \\ Received: September 2017. Accepted : February 2018
}

\begin{abstract}
$A$ set $S$ of a connected graph $G$ of order $n$ is called a double monophonic set of $G$ if for every pair of vertices $x, y$ in $G$ there exist vertices $u, v$ in $S$ such that $x, y$ lie on a $u-v$ monophonic path. The double monophonic number $\operatorname{dm}(G)$ of $G$ is the minimum cardinality of a double monophonic set. A double monophonic set $S$ in a connected graph $G$ is called a minimal double monophonic set if no proper subset of $S$ is a double monophonic set of $G$. The upper double monophonic number of $G$ is the maximum cardinality of a minimal double monophonic set of $G$, and is denoted by $\mathrm{dm}^{+}(G)$. Some general properties satisfied by upper double monophonic sets are discussed. It is proved that for a connected graph $G$ of order $n, d m(G)=n$ if and only if $d m^{+}(G)=n$. It is also proved that $d m(G)=n-1$ if and only if $d m^{+}(G)=n-1$ for a non-complete graph $G$ of order $n$ with a full degree vertex. For any positive integers $2 \leq a \leq b$, there exists a connected graph $G$ with $d m(G)=a$ and $d m^{+}(G)=b$.
\end{abstract}

Key words : Double monophonic set, double monophonic number, upper double monophonic set, upper double monophonic number.

2010 Mathematics Subject Classification : 05C12. 


\section{Introduction}

By a graph $G=V, E$ we mean a finite, undirected connected graph without loops or multiple edges. The order and size of $G$ are denoted by $n$ and $m$, respectively. For basic graph theoretic terminology we refer to [3]. The distance $d(x, y)$ is the length of the shortest $x-y$ path in $G$. Any $x-y$ path of length $d(x, y)$ is called an $x-y$ geodetic. A subset $S$ of $v$ is called a geodetic set of the graph $G$ if every vertex $x$ of $G$ lies on a $u-v$ geodesic for some vertices $u, v$ in $S$. A geodetic set of minimum cardinality is a minimum geodetic set. The cardinality of a minimum geodetic set is the geodetic number of $G$ and is denoted by $g(G)$. The geodetic number of a graph was introduced and studied in $[1,2,4]$. Denote by $I[x, y]$ the set of all vertices lying on some $x-y$ geodesic of $G$. A vertex $v$ in a connected graph $G$ is called weak extreme if there exists a vertex $u$ in $G$ such that $u$, $v \in I[x, y]$ for a pair of vertices $x, y$ in $G$, then $v=x$ or $v=y$. It is easy to see that each extreme vertex of a graph is weak extreme. For the graph $G$ in Figure 1.1, it is clear that the pair $v_{2}, v_{5}$ lies only on the $v_{2}-v_{5}$ geodesic and so $v_{2}$ and $v_{5}$ are weak extreme vertices of $G$. It is easily seen that each vertex of $G$ is weak extreme. Weak extreme vertices are introduced in [6]. A chord of a path $P$ is an edge joining two non-adjacent vertices of $P$. A path $P$ is called monophonic if it is a chordless path. A subset $S$ of $v$ is called a monophonic set of $G$ if every vertex $v$ of $G$ lies on a $x-y$ monophonic path for some vertices $x$ and $y$ in $S$. The minimum cardinality of a monophonic set of $G$ is called the monophonic number of $G$ and is denoted by $m(G)$. Let $G$ be a connected graph with at least two vertices. A set $S$ of vertices of $G$ is called a double geodetic set of $G$ if for each pair of vertices $x, y$ in $G$, there exist vertices $u, v$ in $S$ such that $x, y$ lie on a $u-v$ geodesic. The double geodetic number $d g(G)$ of $G$ is the minimum cardinality of a double geodetic set. Any double geodetic set of cardinality $d g(G)$ is called $d g$-set of $G$. The double geodetic number of a graph was introduced and studied in [6]. A set $S$ of vertices of $G$ is called a double monophonic set of $G$ if for each pair of vertices $x, y$ in $G$ there exist vertices $u, v$ in $S$ such that $x, y$ lie on a $u-v$ monophonic path. The double monophonic number $\operatorname{dm}(G)$ of $G$ is the minimum cardinality of a double monophonic set. Any double monophonic set of cardinality $\operatorname{dm}(G)$ is called a $d m$-set. The double monophonic number of a graph was introduced and studied in [7]. A double geodetic set $S$ in a connected graph $G$ is called a minimal double geodetic set if no proper subset of $S$ is a double geodetic set of $G$. The upper double geodetic number $d g^{+}(G)$ of $G$ is the maximum cardinality of a minimal 
double geodetic set of $G$. The upper double geodetic number of a graph was introduced and studied in [5]. The following theorems will be used in the sequel.

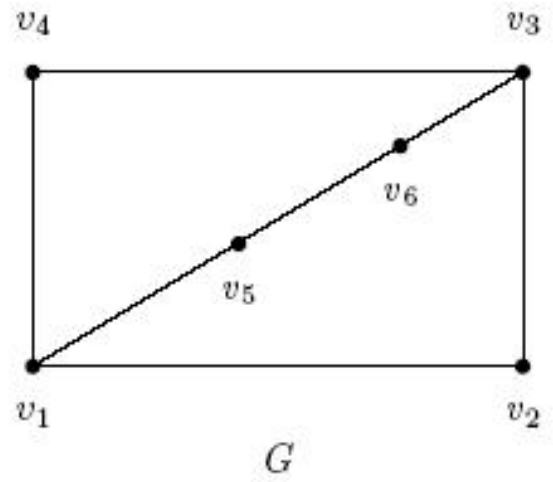

Figure 1.1

A graph with all its vertices weak extreme

Theorem 1.1. [7] Each extreme vertex of a connected graph $G$ belongs to every double monophonic set of $G$. In particular, if the set of all extreme vertices of $G$ is a double monophonic set, then it is the unique minimum double monophonic set of $G$.

Theorem 1.2. [7] Let $G$ be a connected graph with a cut-vertex $v$. Then each double monophonic set of $G$ contains at least one vertex from each component of $G-v$.

Theorem 1.3. [7] No cut-vertex of a connected graph $G$ belongs to any minimum double monophonic set of $G$.

Theorem 1.4. [7] For the complete bipartite graph $G=K_{m, n}(2 \leq m \leq n)$, $d m(G)=\min \{m, n\}$.

\section{Upper double monophonic number of a graph}

Definition 2.1. Let $G$ be a connected graph with at least two vertices. A double monophonic set $S$ in a connected graph $G$ is a minimal double 
monophonic set if no proper subset of $S$ is a double monophonic set of $G$. The upper double monophonic number of a graph $G$ is the maximum cardinality of a minimal double monophonic set of $G$, denoted by $d m^{+}(G)$.

Example 2.2. For the graph $G$ in Figure 2.1, $S=\left\{v_{2}, v_{4}\right\}$ is the only double monophonic set of $G$ so that $\operatorname{dm}(G)=2$. The sets $S_{1}=\left\{v_{1}, v_{2}, v_{4}\right\}$, $S_{2}=\left\{v_{1}, v_{3}, v_{5}\right\}, S_{3}=\left\{v_{2}, v_{3}, v_{4}\right\}$ and $S_{4}=\left\{v_{2}, v_{4}, v_{5}\right\}$ are the only double monophonic sets of cardinality 3 . Hence $S_{2}=\left\{v_{1}, v_{3}, v_{5}\right\}$ is the only minimal double monophonic set of cardinality 3 . It is easily verified that all 4-element subsets are double monophonic, and none of them is minimal. Thus $d m^{+}(G)=3$.

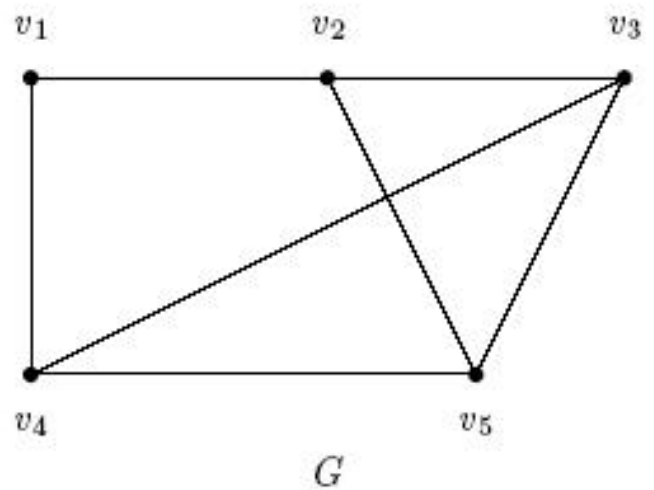

Figure 2.1

It is clear that every minimum double monophonic set of $G$ is a minimal double monophonic set of $G$. The converse need not be true. For the graph $G$ given in Figure 2.1, $S_{2}=\left\{v_{1}, v_{3}, v_{5}\right\}$ is a minimal double monophonic set, and not a minimum double monophonic set.

Theorem 2.3. For any connected graph $G$ of order $n, 2 \leq d m(G) \leq$ $d m^{+}(G) \leq n$.

Proof. Since every double monophonic set contains at least two vertices, it follows that $d m(G) \geq 2$. Also every minimal double monophonic set is a double monophonic set so that $d m(G) \leq d m^{+}(G)$. Thus $2 \leq d m(G) \leq$ $d m^{+}(G) \leq n$. 
The bounds in Theorem 2.3 are sharp. For any non-trivial path $P, d m(P)=$ 2. By Theorems 1.1 and 1.2, it is clear that $d m(T)=d m^{+}(T)$ for any tree $T$ and $d m^{+}\left(K_{n}\right)=n(n \geq 2)$. Further, all the inequalities in the theorem are strict. For a complete bipartite graph $G=K_{r}, s(3 \leq r \leq s), d m(G)=$ $r, d m^{+}(G)=s$ and $n=r+s$.

Corollary 2.4. For any connected graph $G, d m(G)=n$ if and only if $d m^{+}(G)=n$.

Proof. If $d^{+}(G)=n$, then the vertex set $v$ is the only minimal double monophonic set of $G$. Hence it follows that $v$ is the only minimum double monophonic set of $G$ so that $d m(G)=n$. If $d m(G)=n$, then the result follows from Theorem 2.3

Corollary 2.5. If $G$ is a connected graph of order $n$ with $d m(G)=n-1$, then $d m^{+}(G)=n-1$.

Proof. Since $d m(G)=n-1$, it follows from Theorem 2.3 that $d m^{+}(G)=n$ or $d m^{+}(G)=n-1$. It follows from Corollary 2.4 that $d m^{+}(G)=n-1$.

As a consequence of this theorem, the following corollary is clear.

Corollary 2.6. For the complete graph $G=K_{n},(n \geq 2), d m^{+}(G)=n$.

Remark 2.7. It is proved in [6] that every double geodetic set of a connected graph $G$ contains all the weak extreme vertices of $G$. This result is not true for the case of a double monophonic set. That is, a double monophonic set of a connected graph need not contain all the weak extreme vertices of $G$. For the graph $G$ in Figure 1.1, all the vertices are weak extreme. The set $S=v_{1}, v_{3}$ is a double monophonic set. Thus $d m(G)=2$. However, the vertices $v_{2}, v_{4}, v_{5}, v_{6}$ do not belong to $S$. We now compute the upper double monophonic number of $G$. Clearly,

$S_{1}=\left\{v_{1}, v_{3}\right\}, S_{2}=\left\{v_{1}, v_{6}\right\}$ and $S_{3}=\left\{v_{3}, v_{5}\right\}$ are the only three double monophonic sets, so that $\operatorname{dm}(G)=2$. It is easily verified that the sets $T_{1}=\left\{v_{1}, v_{2}, v_{3}\right\}, T_{2}=\left\{v_{1}, v_{2}, v_{6}\right\}, T_{3}=\left\{v_{1}, v_{3}, v_{4}\right\}, T_{4}=\left\{v_{1}, v_{3}, v_{5}\right\}$, $T_{5}=\left\{v_{1}, v_{3}, v_{6}\right\}, T_{6}=\left\{v_{1}, v_{4}, v_{6}\right\}, T_{7}=\left\{v_{1}, v_{5}, v_{6}\right\}, T_{8}=\left\{v_{2}, v_{3}, v_{5}\right\}$, 
$T_{9}=\left\{v_{2}, v_{4}, v_{5}\right\}, T_{10}=\left\{v_{2}, v_{4}, v_{6}\right\}, T_{11}=\left\{v_{3}, v_{4}, v_{5}\right\}$ and $T_{12}=\left\{v_{3}, v_{5}, v_{6}\right\}$ are double monophonic sets of $G$. Out of these, $T_{9}=\left\{v_{2}, v_{4}, v_{5}\right\}$ and $T_{10}=\left\{v_{2}, v_{4}, v_{6}\right\}$ are the only two minimal double monophonic sets. It is verified that all the 4-element sets are double monophonic, and none of them is minimal. It is also verified that all 5-element sets are double monophonic, and none of them is minimal. Hence for this graph $m(G)=2$, $d m(G)=2$ and $d m^{+}(G)=3$.

A vertex in a graph $G$ of order $n$ is called a full degree vertex if its degree is $n-1$. The monophonic closed interval $I_{m}[x, y]$ consists of all vertices lying on some $x-y$ monophonic path of $G$.

Theorem 2.8. Let $G$ be a non-complete connected graph. Then a full degree vertex does not belong to any minimal double monophonic set of $G$.

Proof. Let $S$ be a minimal double monophonic set of $G$ containing a full degree vertex $v_{1}$. Let $S^{\prime}=S-\left\{v_{1}\right\}$. We claim that $S^{\prime}$ is double monophonic set of $G$. Let $x, y \in v$.

Case 1. $x, y \in S$. If $v_{1} \neq x, y$, then $x, y \in S^{\prime}$ and so $S^{\prime}$ is double monophonic set of $G$. So assume that $x=v_{1}$. If $y$ is not a full degree vertex, then there exists $y^{\prime} \neq y$ such that $y$ and $y^{\prime}$ are non-adjacent and so $x, y \in I_{m}\left[y^{\prime}, y\right]$ with $y^{\prime}, y \in S^{\prime}$. Now, if $y$ is a full degree vertex, then since the subgraph induced by $S$ is not complete, there exist non-adjacent vertices $y^{\prime}, y^{\prime \prime}$ in $S$ such that $x, y \in I_{m}\left[y^{\prime}, y^{\prime \prime}\right]$. Thus $S^{\prime}$ is a double monophonic set of $G$, which is a contraction to $S$ a minimal double monophonic set.

Case 2. $x \notin S$ or $y \notin S$. Since $S$ is a double monophonic set, there exists $u, v \in S$ such that $x, y \in I_{m}[u, v]$. Since $v_{1}$ is a full degree vertex, it follows that $u \neq v_{1}$ and $v \neq v_{1}$. Thus $u, v \in S^{\prime}$ and so $S^{\prime}$ is a double monophonic set of $G$, which is again a contradiction to $S$ a minimal double monophonic set of $G$. Thus the proof is complete.

Theorem 2.9. Let $G$ be a non-complete graph of order $n$ with a full degree vertex $v$. Then $d m^{+}(G)=n-1$ if and only if $d m(G)=n-1$. 
Proof. If $d m(G)=n-1$, then by Corollary $2.5, d m^{+}(G)=n-1$. Let $d m^{+}(G)=n-1$. Let $S$ be a minimal double monophonic set of cardinality $n-1$. By Theorem 2.8, $v \notin S$. Suppose that $d m(G) \leq n-2$. Let $S^{\prime}$ be a minimum double monophonic set of $G$. Then it follows from Theorem 2.8 that $v \notin S^{\prime}$ and $S^{\prime} \subseteq S$, which is a contradiction to $S$ a minimal double monophonic set of $G$. Hence $d m(G)=n-1$.

Theorem 2.10. Let $G$ be a connected graph with a cut vertex $v$. Then every minimal double monophonic set of $G$ contains at least one vertex from each component of $G-v$.

Proof. This follows from Theorem 1.2

Theorem 2.11. No cut vertex of a connected graph $G$ belongs to any minimal double monophonic set of $G$.

Proof. Let $S$ be any minimal double monophonic set of $G$. Suppose that $S$ contains a cut vertex $w$ of $G$. Let $G_{1}, G_{2}, \ldots, G_{k}(k \geq 2)$ be the components of $G-w$. Let $S_{1}=S-\{w\}$. We show that $S_{1}$ is a double monophonic set of $G$. Let $u, v$ be any two vertices of $G$. Since $S$ is a double monophonic set, there exist $x, y \in S$ such that $u, v \in I_{m}[x, y]$. If $w \notin\{x, y\}$, then $x, y \in S_{1}$ and so $S_{1}$ is a double monophonic set of $G$, which is a contradiction to the minimality of $S$. Now, assume that $w \in\{x, y\}$, say $w=x$. Assume without loss of generality that $y$ belongs to $S_{1}$. By theorem 2.10, we can choose a vertex $z$ in $G_{l}(l \neq 1)$ such that $z \in S$. Now, since $w$ is a cut vertex of $G$, it follows that $I_{m}[w, y] \subseteq I_{m}[z, y]$. Hence $u, v \in I_{m}[z, y]$, where $z, y \in S_{1}$ so that $S_{1}$ is a double monophonic set of $G$, which is a contradiction to the minimality of $S$. Thus no cut vertex belongs to any minimal double monophonic set of $G$.

In the following we present the upper double monophonic number of some standard graphs.

Theorem 2.12. For any tree $T$ with $k$ end-vertices $d m(T)=k=d m^{+}(T)$.

Proof. This follows from Theorems 1.1 and 2.11 
Theorem 2.13. For the complete bipartite graph $G=K_{m, n}$,

(i) $d m^{+}(G)=2$ if $m=n=1$.

(ii) $d m^{+}(G)=n$ if $m=1, n \geq 2$.

(iii) $d m^{+}(G)=\max \{m, n\}$ if $m, n \geq 2$.

Proof. Results (i) and (ii) follow from Theorem 2.12. (iii) Let $X$ and $Y$ be the partite sets of $K_{m, n}$. Let $S$ be a double monophonic set of $K_{m, n}$. We claim that $X \subseteq S$ or $Y \subseteq S$. Otherwise, there exist vertices $x, y$ such that $x \in X, y \in Y$ and $x, y \notin S$. It is clear the pair of vertices $x, y$ lie only on the intervals $I_{m}[x, y], I_{m}[x, t]$ and $I_{m}[s, y]$ for some $t \in X$ and $s \in Y$. Therefore $x \in S$ or $y \in S$, which is a contradiction. Thus $X \subseteq S$ or $Y \subseteq S$. Since both $X$ and $Y$ are double monophonic sets of $K_{m, n}$ the result follows.

\section{Theorem 2.14.}

(i) For the cycle $G=C_{n}(n \geq 4), d m(G)=d m^{+}(G)=2$.

(ii) For the wheel $G=W_{1}, n-1, d m(G)=d m^{+}(G)=2$.

(iii) For the graph $G=K_{n}-e, d m^{+}(G)=2$.

Proof. (i) It is clear that any set $S$ of vertices consisting of two nonadjacent vertices is a double monophonic set so that $\operatorname{dm}(G)=2$. Now let $T$ be any double monophonic set of vertices such that $|T| \geq 3$. Then $S$ contains at least two non-adjacent vertices so that $T$ is not minimal. It follows that $d m^{+}(G)=2$.

(ii) Let $v=\left\{v, v_{1}, v_{2}, \ldots, v_{n-1}\right\}$ with $v$ the central vertex and $v_{1}, v_{2}, \ldots, v_{n-1}$ the cycle $C_{n-1}$. Let $S$ be any set consisting of two non-adjacent vertices on the cycle $C_{n-1}$. It is clear that $S$ is a double monophonic set of $G$ so that $d m(G)=2$. Now, let $T$ be any double monophonic set of vertices such that $|T| \geq 3$. Then $S$ contains at least two non-adjacent vertices so that $T$ is not minimal. It follows that $d m^{+}(G)=2$.

(iii) Let $e$ be the edge $e=u v$. Then $u$ and $v$ are the only extreme vertices of $G$ and it is clear that $S=\{u, v\}$ is a double monophonic set so that $d m(G)=2$. Let $T$ be any double monophonic set such that $|T| \geq 3$. Since $u$ and $v$ are extreme vertices, by Theorem 1.1, $u, v \in T$ so that $T$ is not minimal. Hence $d m^{+}(G)=2$.

The following theorem is a realization result with regard to Theorem 2.3 
Theorem 2.15. For any positive integers $2 \leq a \leq b$, there exists a connected graph $G$ such that $d m(G)=a$ and $d m^{+}(G)=b$.

Proof. For $a=b$, it follows from Theorem 2.12 that $d m(G)=d m^{+}(G)=a$, where $G=K_{1, a}$. For $a<b$, it follows from Theorem 2.13 that $d m(G)=a$ and $d m^{+}(G)=b$, where $G=K_{a}, b$.

\section{Acknowledgements}

The authors are thankful to the referee for his valuable comments.

\section{References}

[1] F. Buckley and F. Harary, Distance in Graphs, Addison Wesley, Redwood city, CA, (1990).

[2] G. Chartrand, F. Harary and P. Zhang, On the geodetic number of a graph, Networks 39, pp. 1-6, (2002).

[3] F. Harary, Graph Theory, Addision Wesley, U.S.A.,(1969).

[4] F. Harary, E. Loukakis and C. Tsouros, The geodetic number of a graph, Math. Comput. Modeling 17, pp. 89 - 95, (1993).

[5] A. P. Santhakumaran and T. Jebaraj, The upper double geodetic number of a graph,Malaysian Journal of Science 30 (3): 225- 229, (2011).

[6] A. P. Santhakumaran and T. Jebaraj, The double geodetic number of a graph, Discuss. Math. Graph Theory, 32, pp. 109-119, (2012).

[7] A. P. Santhakumaran and T. Venkata Raghu, The double monophonic number of a graph, International Journal of Computational and Applied Mathematics, 11 (1), pp. 21-26, (2016). 


\section{A. P. Santhakumaran}

Department of Mathematics

Hindustan Institute of Technology and Science

Chennai-603 103,

India

e-mail : apskumar1953@gmail.com

and

\section{T. Venkata Raghu}

Department of Applied Sciences and Humanities

Sasi Institute of Technology and Engineering

Tadepalligudem 534 101,

India

e-mail : tvraghu2010@gmail.com 